
\title{
Research S Surate \\ High expression of AFFs is related to prognosis of acute myeloid leukemia
}

\section{xi chen}

Southwest Medical University https://orcid.org/0000-0002-8207-7946

\section{Qin Yan}

Southwest Medical University

\section{Xiang Qin}

The Affiliated Hospital of Southwest Medical University

Jing Liu

Southwest Medical University

\section{Yan Zeng}

Southwest Medical University

\section{Sili Long}

The Affiliated Hospital of Southwest Medical University

\section{Wenjun Liu ( $\square$ wenjun_liu@swmu.edu.cn )}

The Affiliated Hospital of Southwest Medical University https://orcid.org/0000-0002-7205-0598

\section{Research}

Keywords: AFF family genes, acute myeloid leukemia, bioinformatics analysis, prognosis

Posted Date: September 28th, 2021

DOI: https://doi.org/10.21203/rs.3.rs-927123/v1

License: (c) (1) This work is licensed under a Creative Commons Attribution 4.0 International License.

Read Full License 


\title{
High expression of AFFs is related to prognosis of acute myeloid leukemia
}

Xi Chen\#, Qin Yan\#, Xiang Qin, Jing Liu, Yan Zeng, Sili Long, Wenjun Liu*

Department of Pediatrics Hematology, The Affiliated Hospital of Southwest Medical University, Luzhou, Sichuan, 646000, China; Children Hematological Oncology and Birth Defects Laboratory, The Affiliated Hospital of Southwest Medical University, Luzhou, Sichuan, 646000, China; Sichuan Clinical Research Center for Birth Defects, Luzhou, Sichuan, 646000, China

*Correspondence to: Wenjun Liu. Department of Pediatrics Hematology, The Affiliated Hospital of Southwest Medical University, Luzhou, Sichuan, 646000, China; Children Hematological Oncology and Birth Defects Laboratory, The Affiliated Hospital of Southwest Medical University, Luzhou, Sichuan, 646000, China; Sichuan Clinical Research Center for Birth Defects, Luzhou, Sichuan, 646000, China.

\# These authors contributed equally to this work.

\begin{abstract}
Background: AFF family genes (AFF1, AFF2, AFF3, AFF4, AFFs) are closely related to the occurrence, development and prognosis of a variety of tumors, but the relationship between AFFs and acute myeloid leukemia (AML) is still unclear. This study aims to explore the relationship between AFFs and the prognosis of AML by bioinformatics and real time quantitative PCR.

Methods: Analyze the expression of AFFs in AML patients and AML cell lines through ONCOMINE, GEPIA, EMBL-EBI, and CCLE databases, explore the prognostic value of AFFs in AML through the TCGA database,explore Co-expression and functional enrichment analysis of AFFs through the Linkedomics, DAVID and KOBAS database.We collected peripheral blood samples of 24 cases of AML (non-M3 subtype) and 8 cases of benign individuals, and detected the mRNA expression level of AFFs by real-time fluorescent quantitative PCR (clinical data comes from hospitalized medical records).
\end{abstract}

Results: The results of multiple data sets showed that the mRNA transcription level of AFF1, AFF2, AFF3, and AFF4 in AML was significantly higher than that of 
normal people. Among them, the high expression of AFF1, AFF2, and AFF3 was statistically significant $(\mathrm{p}<0.05)$. Our clinical data showed that the mRNA expression levels of AFF1 and AFF2 in AML patients were higher than those in the control group $(\mathrm{p}<0.05)$. The high expression of AFF1 was significantly negatively correlated with overall survival (OS) $(\mathrm{p}<0.05)$, the high expression of AFF2 was significantly positively correlated with OS $(\mathrm{p}<0.05)$, and the high expression of AFF3 was not correlated with OS $(\mathrm{p}>0.05)$.

Conclusion: AFF1 and AFF2 are highly expressed in AML. The high expression of AFF1 is significantly negatively correlated with OS, and the high expression of AFF2 is significantly positively correlated with OS, which may become a new target for treatment.

Keywords: AFF family genes; acute myeloid leukemia; bioinformatics analysis; prognosis;

\section{Background}

Acute myeloid leukemia (AML) is a malignant disease of myeloid hematopoietic stem/progenitor cells, characterized by abnormal proliferation of primitive and naive myeloid cells in the bone marrow and peripheral blood [1], it's prognosis closely related to cytogenetics and molecular abnormalities, such as NPM1 (nucleophosmin 1, NPM1), CEBPA (CCAAT/enhancer binding protein alpha, CEBPA), FLT3 (Fms-like tyrosine kinase 3, FLT3)-ITD (internal tandem duplication, ITD), P53, and chromosome aneuploidy mutations, etc. [2]The prognosis is very different [3]. Although recent research on FLT3 mutations as a therapeutic target has made major breakthroughs in the treatment of AML, the morbidity and mortality rates are still high, with an overall five-year survival rate of 20\% [4-6]. The five-year survival rate for patients under the age of 60 is $35 \%-40 \%$ while the patients over 60 years is 5\%-15\% [7]. Therefore, finding more therapeutic targets is very important for AML.

The AFF family, namely the AF4 / FMR2 family, is a family of RNA binding proteins (AFF1, AFF2, AFF3, AFF4, AFFs), which are located in the nucleus and act as transcriptional activators to have a positive effect on RNA elongation[8]. The main 
function of AFFs gene is to regulate transcription, which plays an important role in the occurrence and development of a variety of tumors. AFF1 is involved in inhibiting the migration and invasion of serous epithelial ovarian cancer (SEOC) cells [9] and down-regulating indicators related to poor prognosis of lung adenocarcinoma[10]; in leukemia, The protein AF4 encoded by AFF1 acts as a MLL fusion partner. The low expression of MLL-AF4 can induce apoptosis and hinder cell proliferation[11]。 AFF1 is also involved in the KMT2A gene rearrangement in infantile acute lymphoblastic leukemia, and the KMT2A-AFF1 rearrangement is the independent risk factors for prognosis[12]. AFF2 is not only related to the prognosis of patients with thymoma[13] and neuroblastoma[14], but also involved in the occurrence and development of non-keratinizing squamous cell carcinoma of the nasal cavity[15]. Recent studies have found that CircAFF2, which is derived from AFF2, acts as an oncogene in gastric cancer. Knockout of circAFF2 can significantly inhibit cell migration, invasion and proliferation of gastric cancer cells[16]. circ-AFF2 may also be a potential prognostic biomarker in multiple myeloma (MM) [17]. AFF3 plays an important role in anti-tumor necrosis factor (TNF) therapy[18] and participates in the occurrence and development of adrenocortical cancer (Adrenocortical cancer, ACC)[19]. The increase of AFF3 expression in estrogen receptor-positive (ER) breast tumors is associated with a decrease in overall survival[20]. AFF4 is used as a scaffold protein that connects transcription factors and promotes gene transcription through extension and chromatin remodeling[21]. In leukemia cells, AFF4 may become the key to the pathogenesis of leukemia through many Mixed Lineage Leukemia (MLL) partners[22]. However, the expression of AFF family genes in AML and its relationship with prognostic value are not clear. Therefore, this article aims to explore the value of AFF family genes in predicting the prognosis of AML and to provide a basis for clinical intervention and treatment through recognized databases and clinical samples of AML patients.

\section{2 results}




\subsection{AFFs are highly expressed in AML}

2.1.1AFFs are highly expressed in AML （GEPIA and ONCOMINE database )

We used the GEPIA database to compare the transcription levels of AFFs in AML samples and normal samples. The results showed that AFF1, AFF2, and AFF3 were significantly high expressed with statistical significance $(\mathrm{p}<0.05)$, the high expression of AFF4 has no statistically significant ( $\mathrm{p}>0.05)$ (Figure 1).

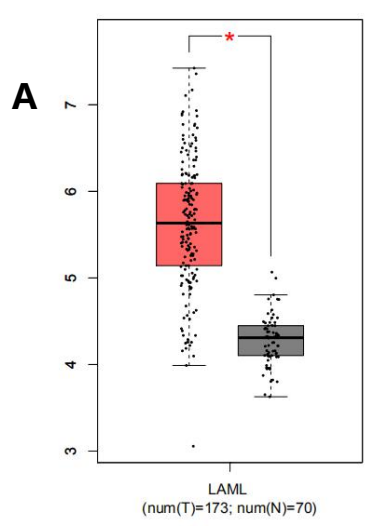

AFF1

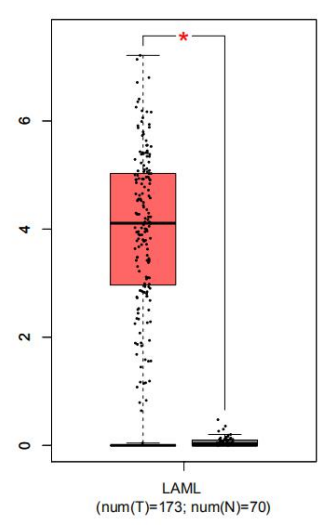

AFF3

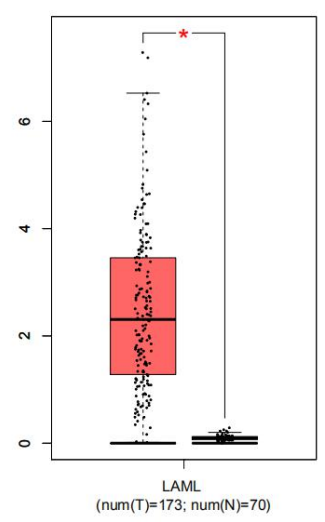

AFF2

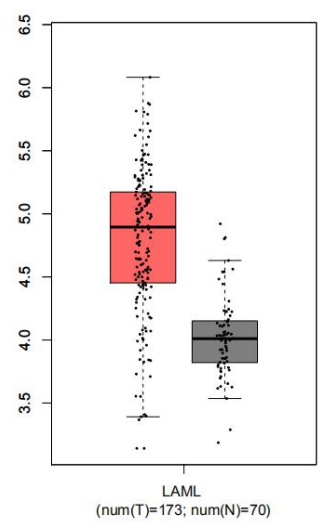

AFF4

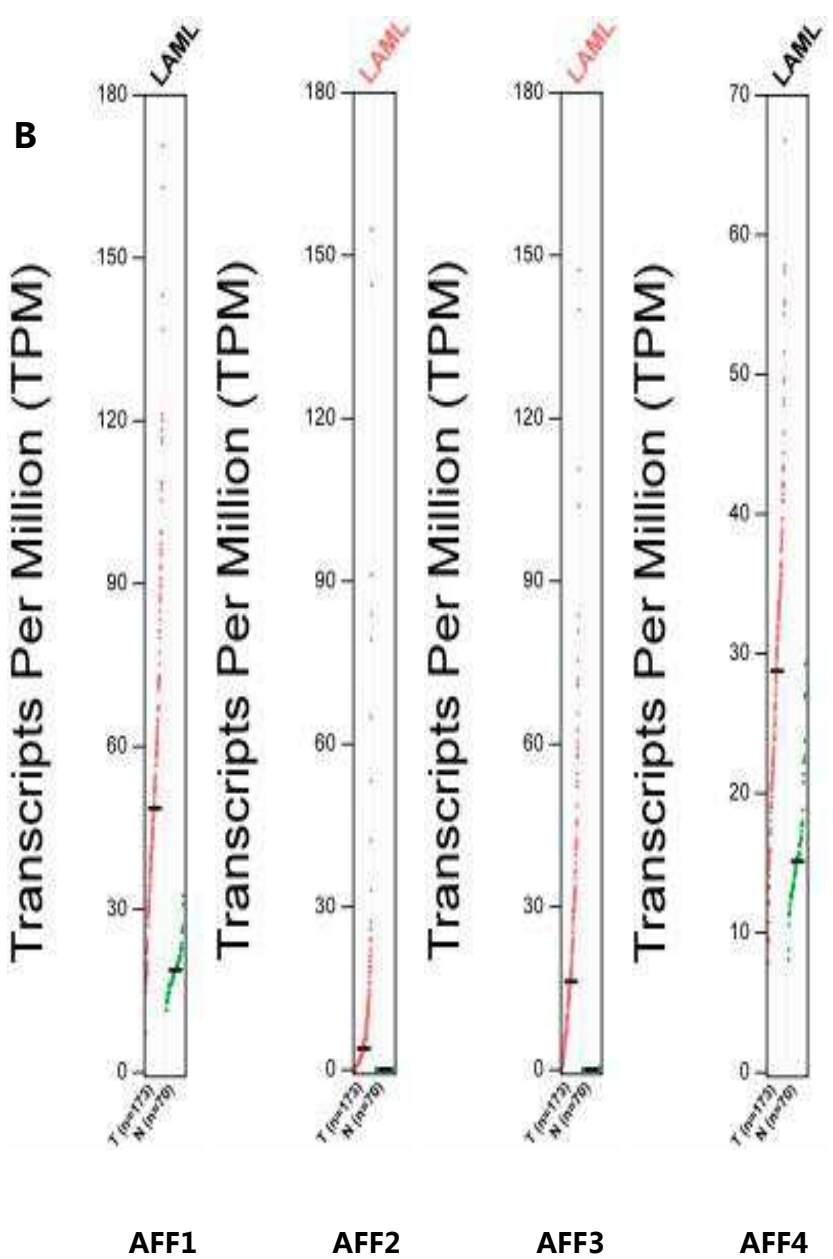

AFF1

AFF2

AFF3

AFF4

Figure 1. The Expression of AFFs in Leukemia (GEPIA) .(AB) The expression levels of AFFs in AML vs normal samples.*: $\mathrm{p}<0.05$. 
Table1.The Signifificant Changes of AFFs Expression in Transcription Level (ONCOMINE Database)

\begin{tabular}{llllll}
\hline \multirow{2}{*}{ GENE } & Types of leukemia vs. Normal Samples & $\begin{array}{l}\text { Fold } \\
\text { Change }\end{array}$ & $\boldsymbol{P}$ Value & $\boldsymbol{t}$-Test & Reference \\
\hline AFF1 & Acute Myeloid Leukemia vs Normal & 4.216 & $1.00 \mathrm{E}-03$ & 4.752 & Stegmaier Leukemia \\
AFF2 & Acute Myeloid Leukemia vs Normal & 2.646 & $3.19 \mathrm{E}-04$ & 4.500 & Stegmaier Leukemia \\
AFF3 & Acute Myeloid Leukemia vs Normal & 1.628 & $4.40 \mathrm{E}-02$ & 1.902 & Stegmaier Leukemia \\
AFF4 & Acute Myeloid Leukemia vs Normal & 1.855 & $8.84 \mathrm{E}-05$ & 5.233 & Stegmaier Leukemia \\
\hline
\end{tabular}

By using the ONCOMINE database, we compared the transcript levels of AFFs in AML and normal samples. ONCOMINE's research results showed that AFF1, AFF2, AFF3, and AFF4 are highly expressed in leukemia (table1) $(\mathrm{P}<0.05)$. In the Stegmaier Leukemia data set, the mRNA transcription level of AFF1 was 4.216 times that of normal people $(\mathrm{P}<0.05)$, the mRNA transcription level of AFF2 was 2.646 times that of normal people $(\mathrm{P}<0.05)$, the transcription level of AFF3 was 1.628 times that of normal people $(\mathrm{P}<0.05)$, The transcription level of AFF4 was 1.855 times that of normal people $(\mathrm{P}<0.05)$.

\subsubsection{Transcription level of AFFs in clinical cases}

We analyzed the mRNA expression levels of 24 AML patients (non-M3 type) in our hospital by real-time fluorescent quantitative PCR. The results showed that the expressions of AFF1 ( $\mathrm{p}=0.006)$, AFF2 $(\mathrm{p}=0.021)$, and AFF4 $(\mathrm{p}=0.014)$ in AML samples were significantly higher than those in the control group (Figure 2) and the high expression of AFF3 has no statistically significant $(p=0.084)$. Further analysis of the post-treatment remission of patients in the AFF high expression group and AFF low expression group (Table 2), we found that there was no significant difference in the expression of AFF family genes between different genders, ages, prognosis, and complete remissions $(\mathrm{P}>0.05))$.the relationship between the expression of AFF family genes and gene mutations and fusion genes has no statistically significant $(\mathrm{P}>0.05)$, which might be related to the small sample size. 

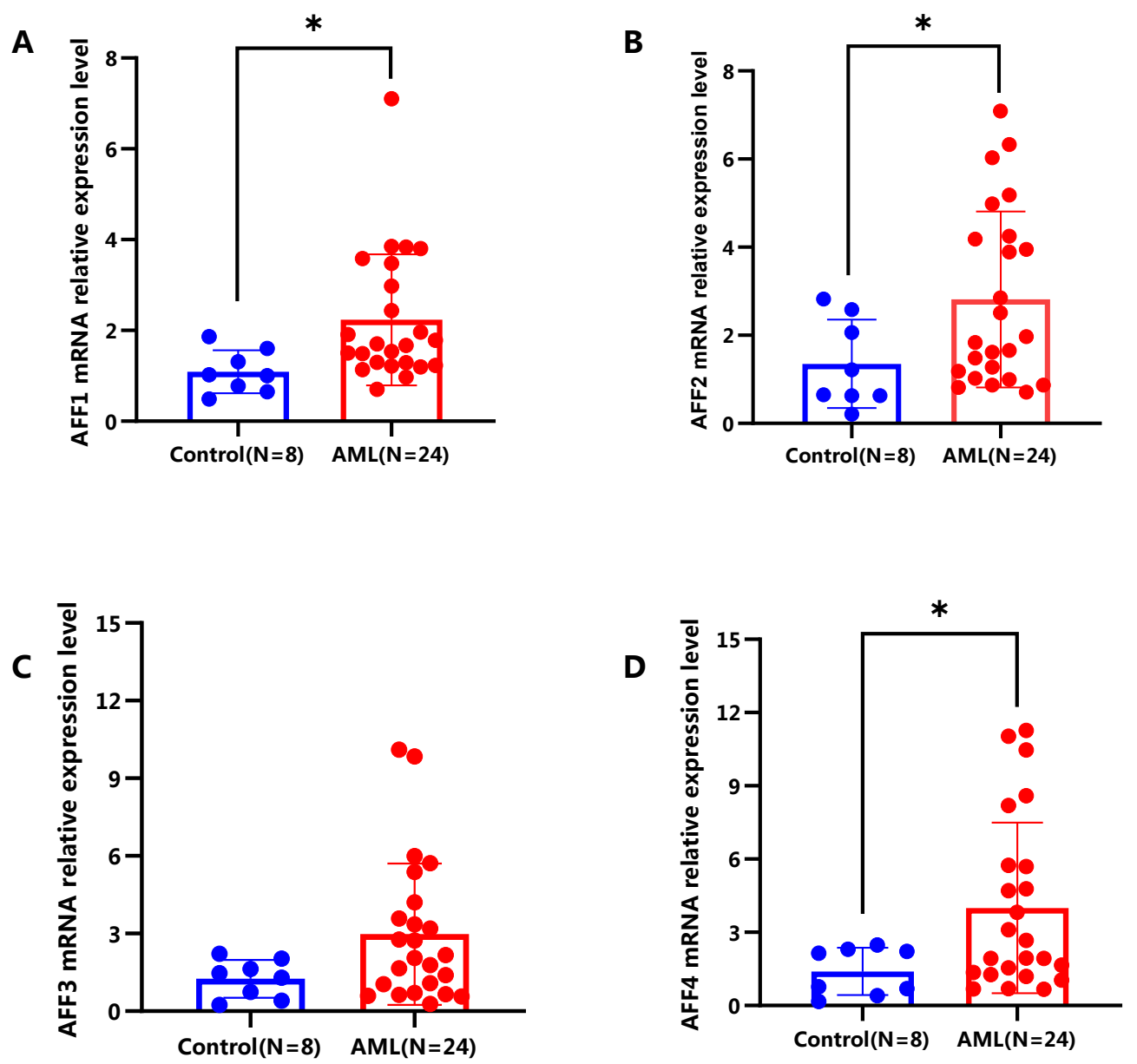

Figure 2. Expression of AFF genes in AML and normal samples.
(A) The expression levels of AFF1 in AML compared with normal samples.
(B) The expression levels of AFF2 in AML compared with normal samples.
(C) The expression levels of AFF3 in AML compared with normal samples.
(D) The expression levels of AFF3 in AML compared with normal samples. *: $p<0.05$. 
Table2 Relationship between baseline characteristics and AFFs mRNA expression. (Fisher exact test)

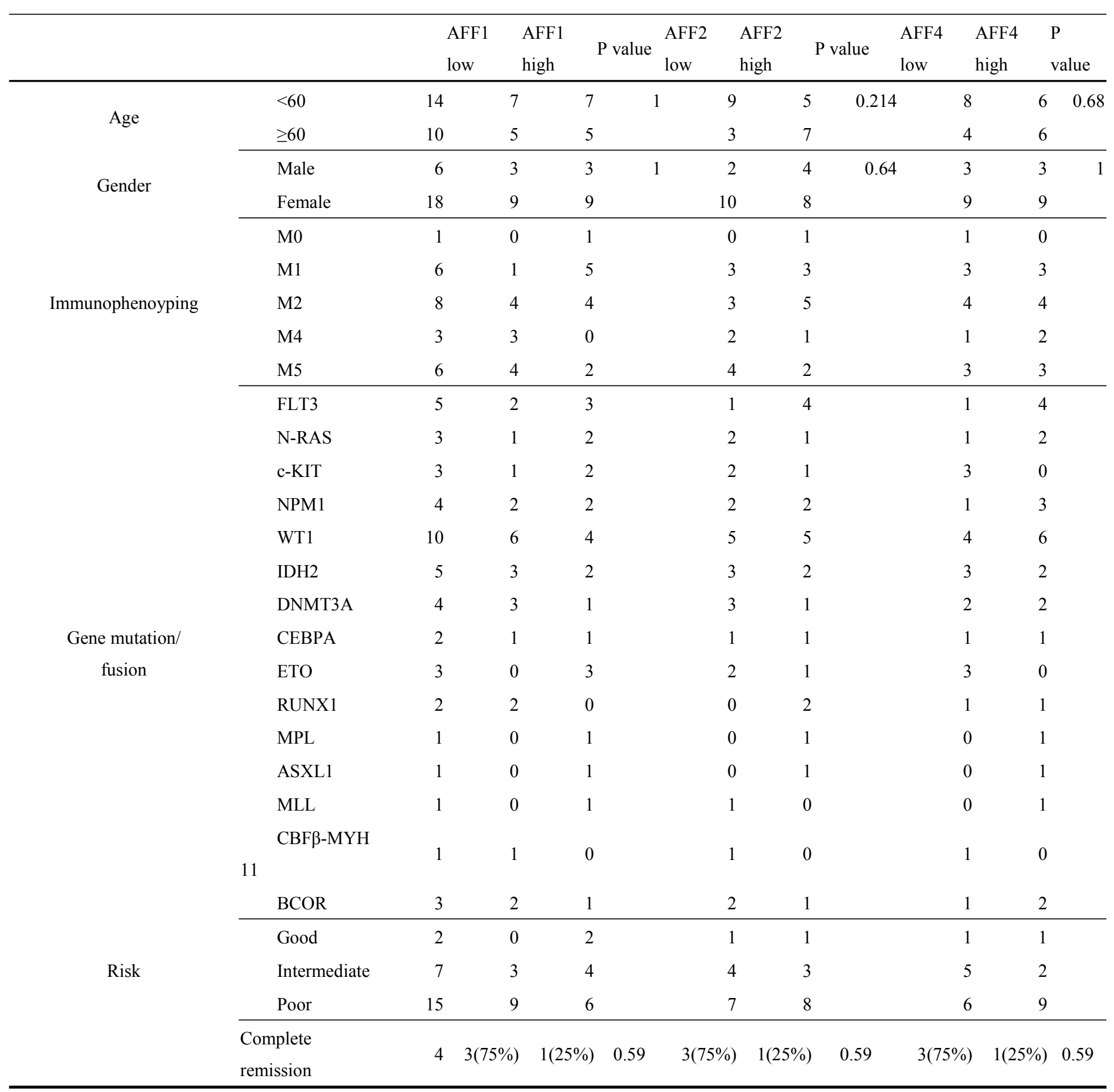




\subsubsection{Transcription level of AFFs in clinical cases}

We analyzed the mRNA expression levels of 24 AML patients (non-M3 type) in our hospital by real-time fluorescent quantitative PCR. The results showed that the expressions of AFF1 $(p=0.006), \operatorname{AFF} 2(p=0.021)$, and AFF4 $(p=0.014)$ in AML samples were significantly higher than those in the control group (Figure 2) and the high expression of AFF3 has no statistically significant $(p=0.084)$. Further analysis of the post-treatment remission of patients in the AFF high expression group and AFF low expression group (Table 2), we found that there was no significant difference in the expression of AFF family genes between different genders, ages, prognosis, and complete remissions $(\mathrm{P}>0.05)$ ).the relationship between the expression of AFF family genes and gene mutations and fusion genes has no statistically significant ( $\mathrm{P}>0.05)$, which might be related to the small sample size.

\subsubsection{Expression of AFFs in different cancer cell lines}

In the CCLE database, the expression level of AFFs in different cancer cells was systematically clarified (Figure $3 \mathrm{ABCD}$ ). We found that AFF1 and AFF4 are highly expressed in acute myeloid leukemia cell lines. Subsequently, we analyzed the expression level of AFFs in acute myeloid leukemia cell lines through EMBL-EBI, and found that AFF1, AFF3, and AFF4 are highly expressed in most acute myeloid leukemia cell lines (Figure 3E).

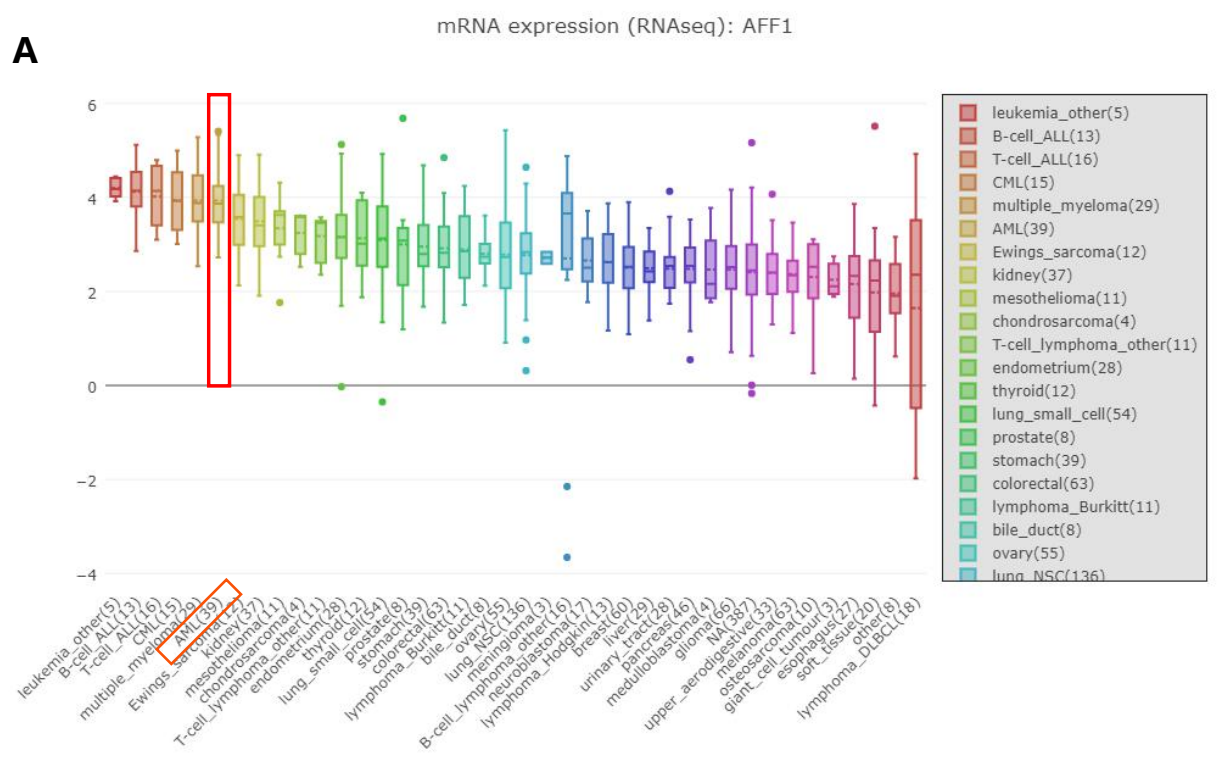




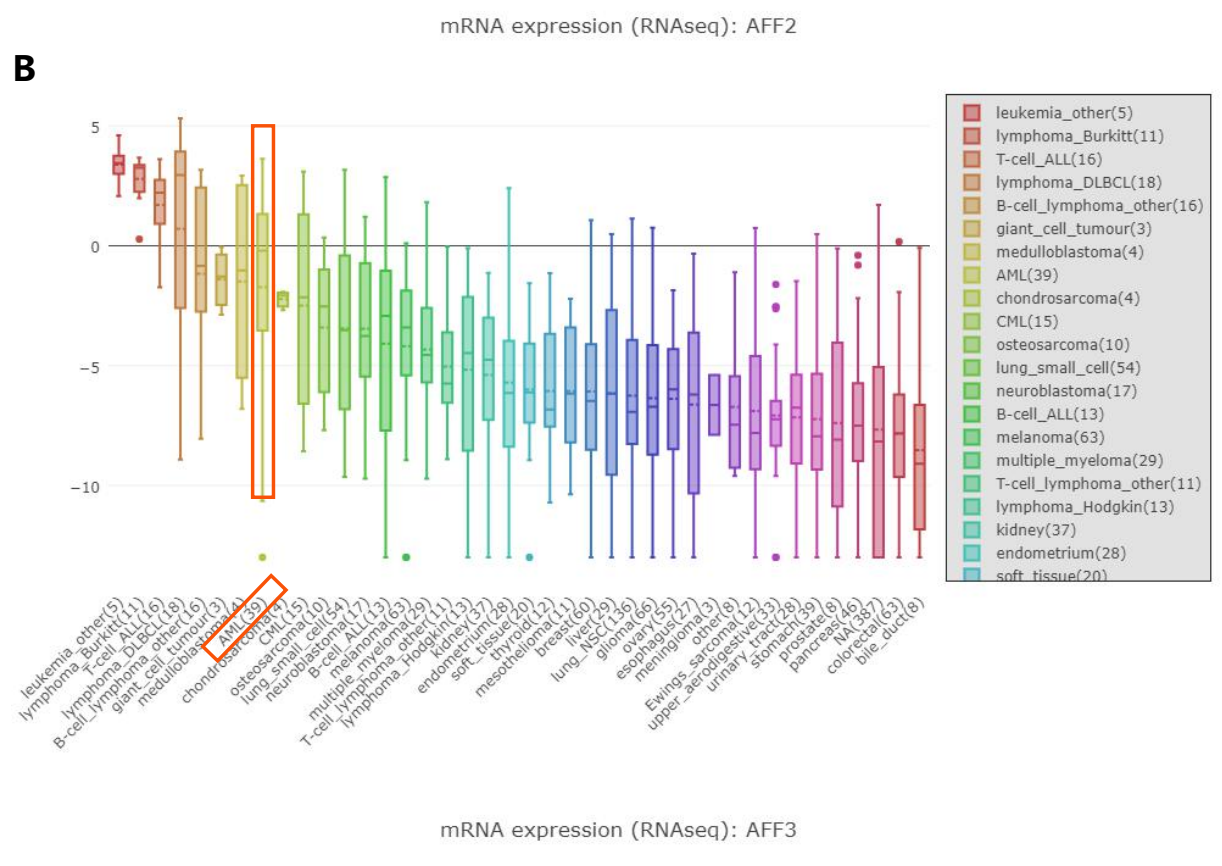

C
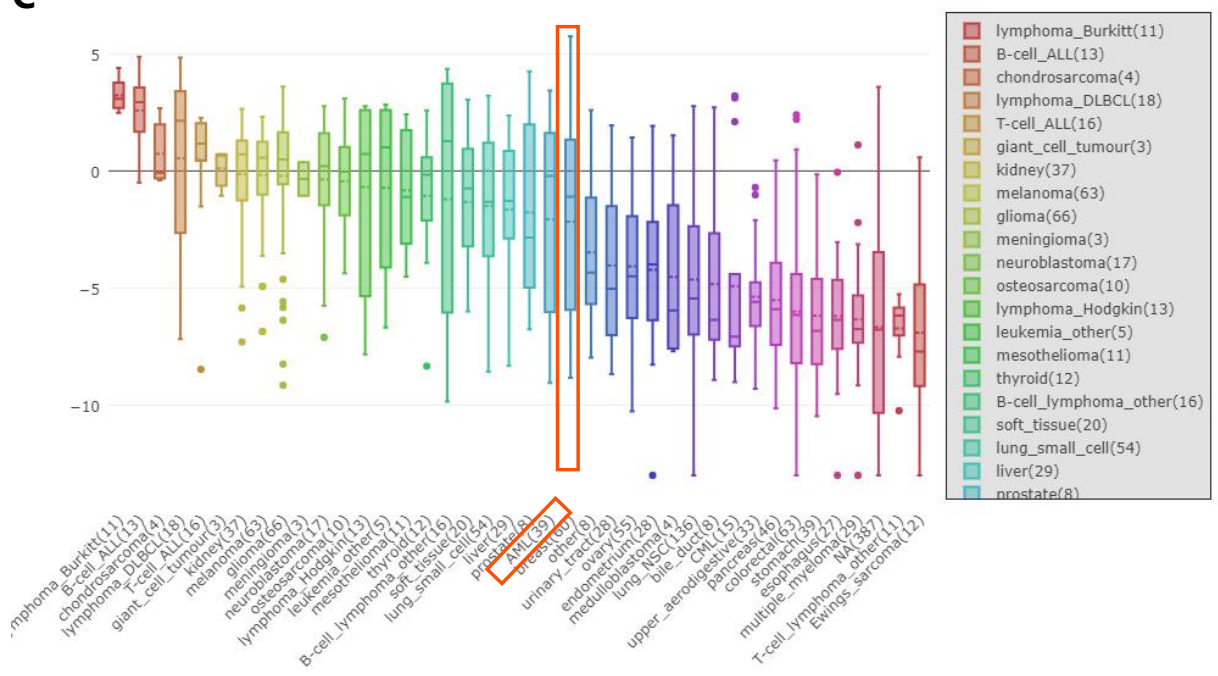

D

mRNA expression (RNAseq): AFF4
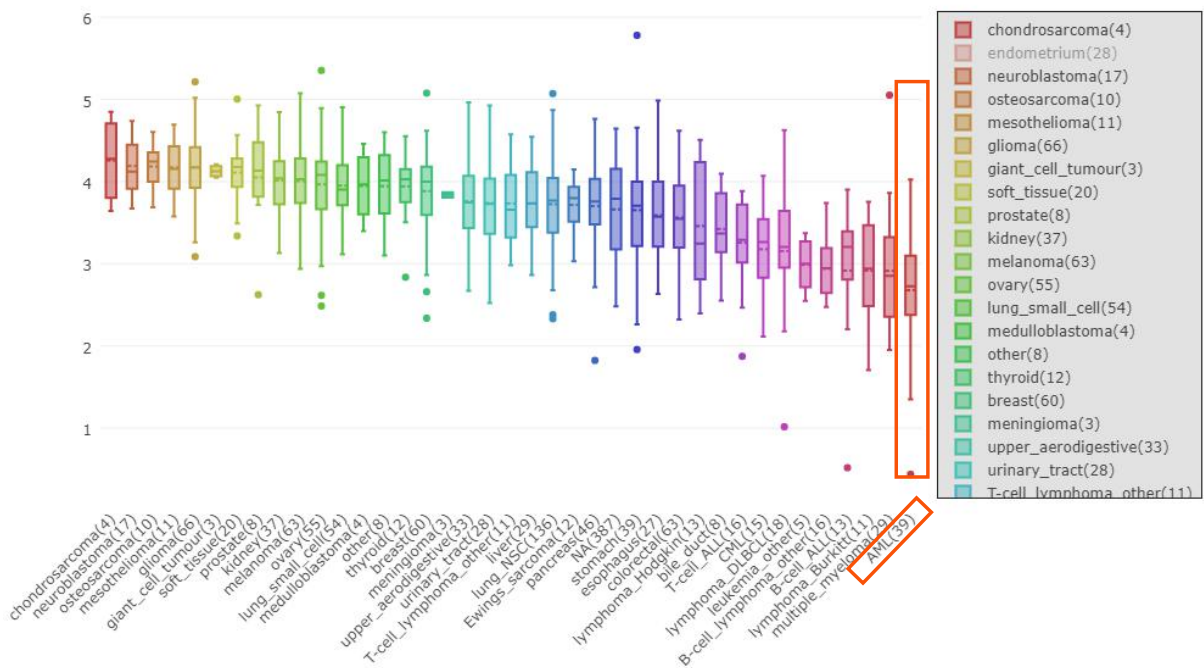


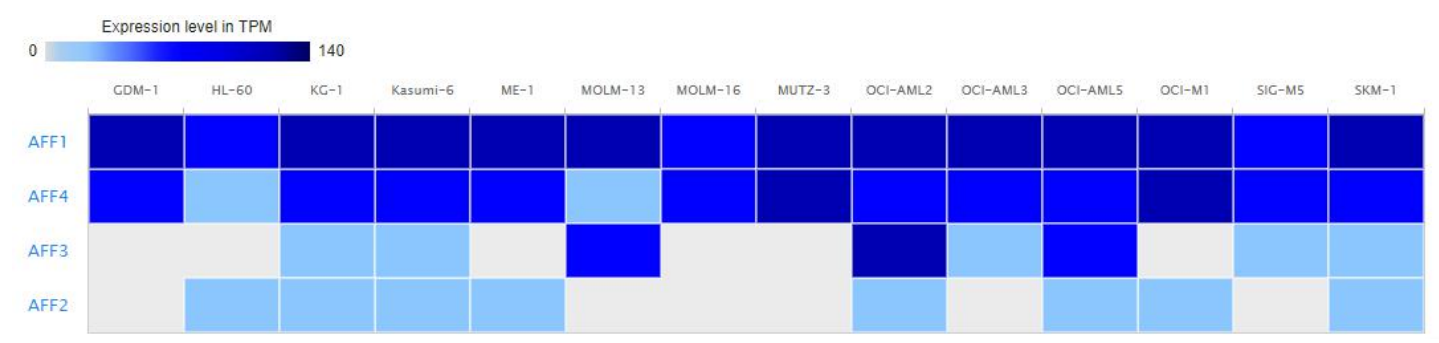

Figure 3 Expression of AFFs in Pan-cancers and AML cell lines (CCLE and EMBL-EBI).

(A) The expression levels of AFF1 in Pan-cancers cell lines by CCLE.

(B) The expression levels of AFF2 in Pan-cancers cell lines by CCLE.

(C) The expression levels of AFF3 in Pan-cancers cell lines by CCLE.

(D) The expression levels of AFF4 in Pan-cancers cell lines by CCLE.

(E) The expression levels of AFFs in AML cell lines by EMBL-EBI.

2.1.4 Expression of AFFs in French-American and British (FAB) Subtypes of AML

In UALCAN, according to the French, American, and British (FAB) classifications of acute leukemia and myelodysplastic syndromes, the expression levels of AFFs are different in different types (Figure 4). The expression of AFF1 in the M3 type was significantly lower than other types, and the differences between M3 and M0 ( $\mathrm{p}=4.36 \mathrm{E}-05), \mathrm{M} 3$ and M1 ( $\mathrm{p}=1.73 \mathrm{E}-02)$ had statistically significant (Figure 4A). The expression of AFF2 in the M3 type was significantly higher than that of other types, and the differences between M3 and M0 ( $p=1.16 \mathrm{E}-04), \mathrm{M} 3$ and M1 $(\mathrm{p}=1.79 \mathrm{E}-04), \mathrm{M} 3$ and M2 (p=2.04E-04), M3 and M4 (p =2.19E-04), M3 and M5 ( $p=1.16 \mathrm{E}-04$ ) had statistically significant (Figure 4B). The expression of AFF3 in the M3 type was significantly lower than that of other types, and the differences between M3 and M0 (p=6.01E-04), M3 and M1 (p=1.62E-02),M3 and M2 (p=8.30E-03), M3 and M4 ( $\mathrm{p}=2.83 \mathrm{E}-04)$, M3 and M5 ( $\mathrm{p}=2.53 \mathrm{E}-02)$ had statistically significant (Figure 4C). The expression of AFF4 in the M3 type was significantly lower than that of M0 $(p=2.36 \mathrm{E}-02)$, and there was no statistically significant between other types and M3 (Figure 4D). 
Expression of AFF1 in LAML based on French American British classification

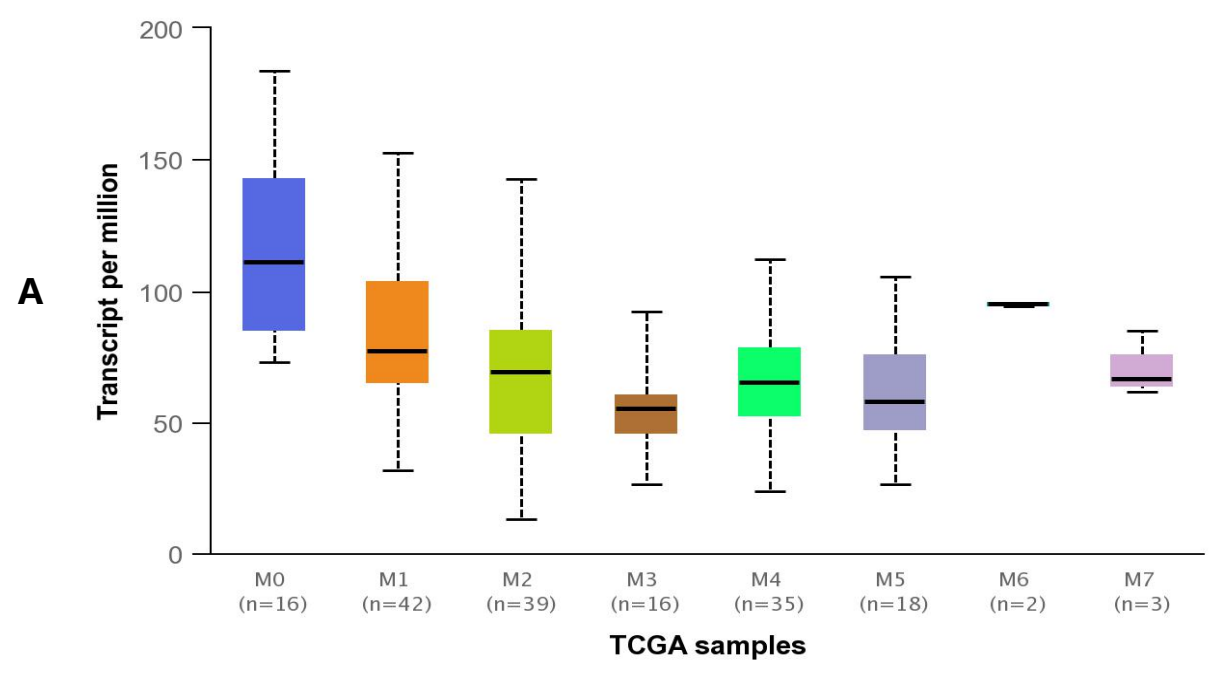

Expression of AFF2 in LAML based on French American British classification

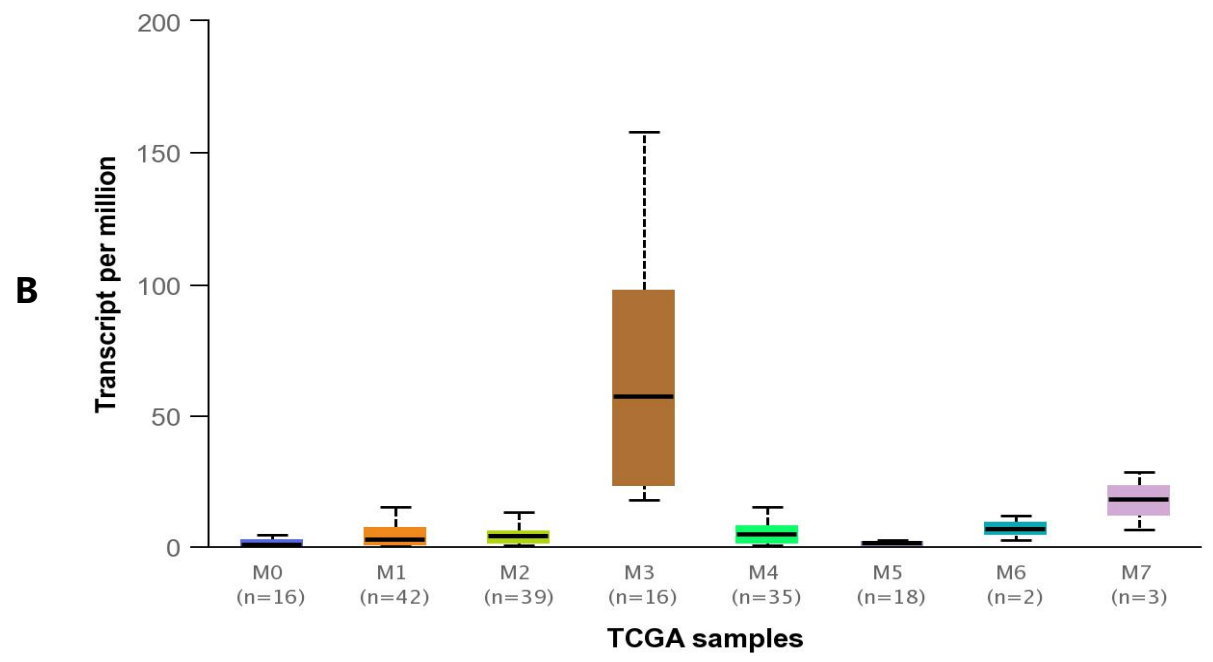

Expression of AFF3 in LAML based on French American British classification

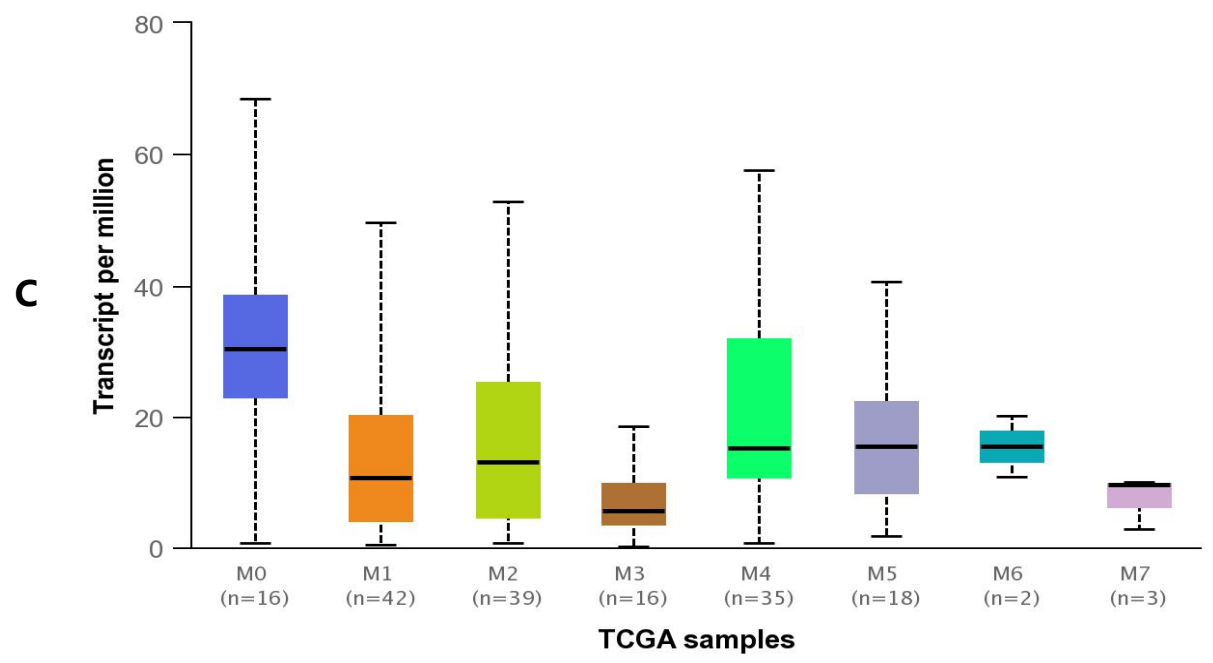




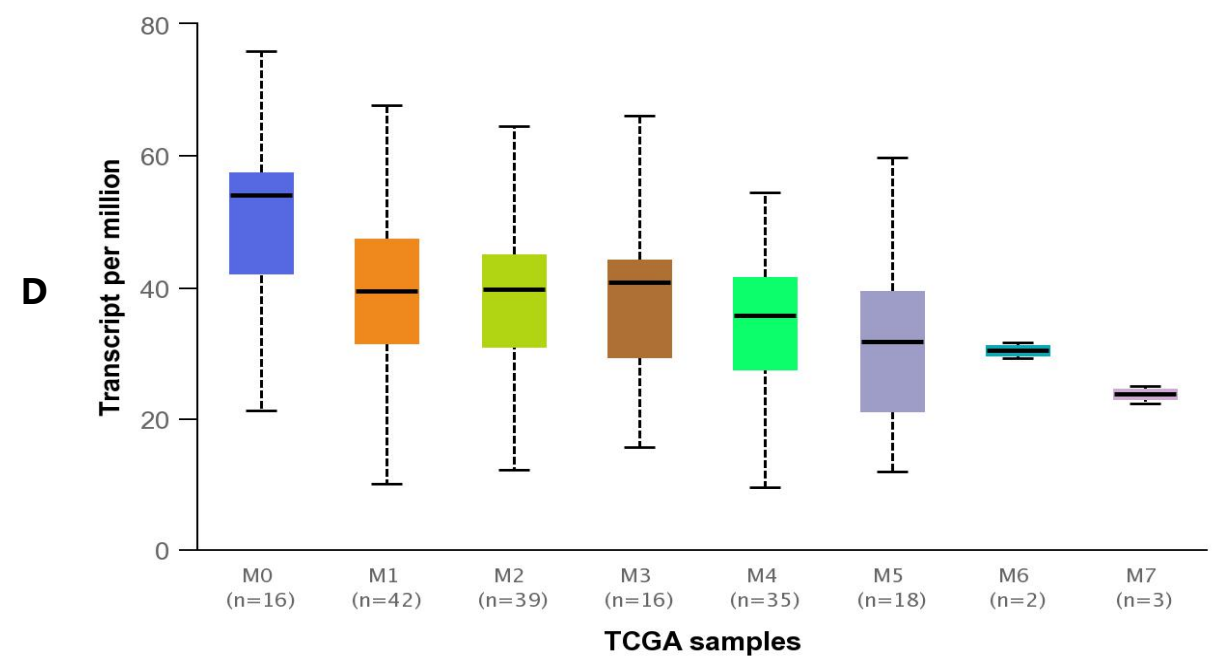

Figure 4 The Expression of AFFs in Leukemia based on French American British classification (UALCAN)

(A)The expression level of AFF1 in AML based on FAB classification.

(B) The expression level of AFF2 in AML based on FAB classification.

(C) The expression level of AFF3 in AML based on FAB classification.

(D) The expression level of AFF4 in AML based on FAB classification.

\subsection{The prognostic value of AFFs in AML}

2.2.1 The prognostic value of AFFs in AML (TCGA)

We downloaded the transcriptome data and clinical data of AML through the TCGA database, and Analyzed the relationship between the expression levels of AFF1, AFF2, AFF3, AFF4 and the prognosis of AML patients(Figure 5).The result showed that the high expression of AFF1 was significantly negatively correlated with the overall survival $(\mathrm{OS})$ of AML $(\mathrm{P}<0.05)$, while the high expression of AFF2 was significantly positively correlated with the OS of AML $(\mathrm{P}<0.05)$, and AFF3 and AFF4 had no prognostic value in AML $(\mathrm{P}>0.05)$. 
A

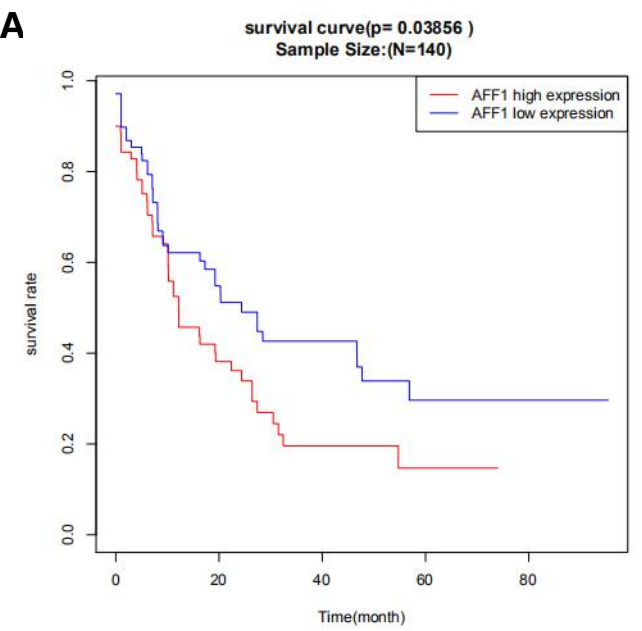

C

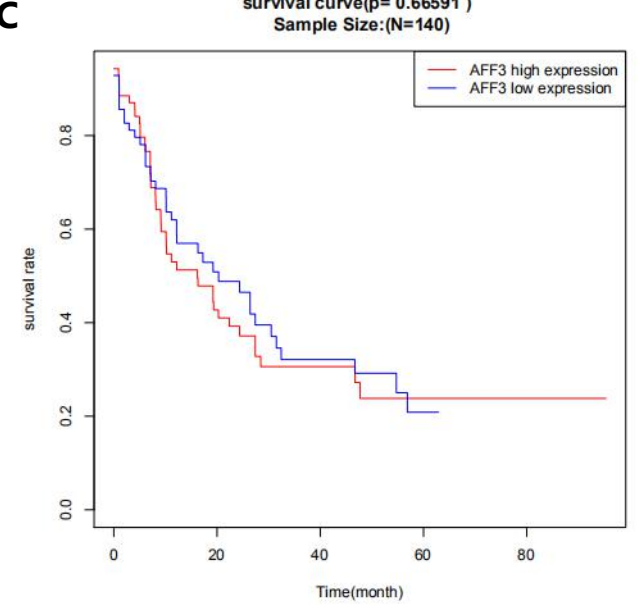

B $\quad \begin{aligned} & \text { survival curve }(p=0.00357) \\ & \text { Sample Size: }(N=140)\end{aligned}$

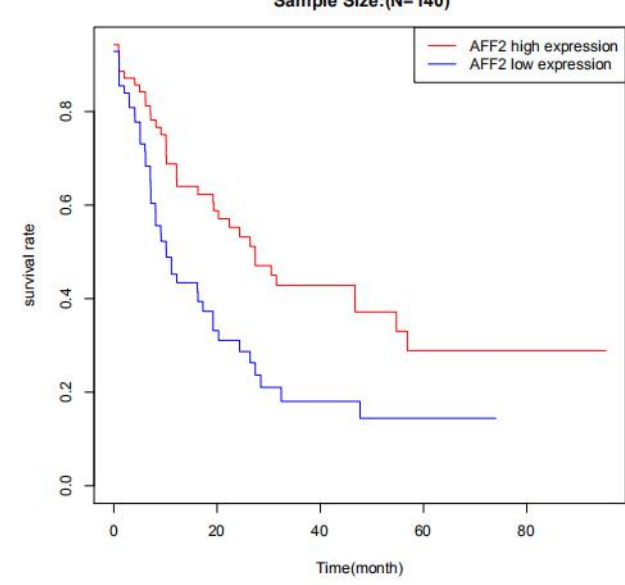

D Survival curve $(p=0.30255)$
Sample Size: $(\mathrm{N}=140)$

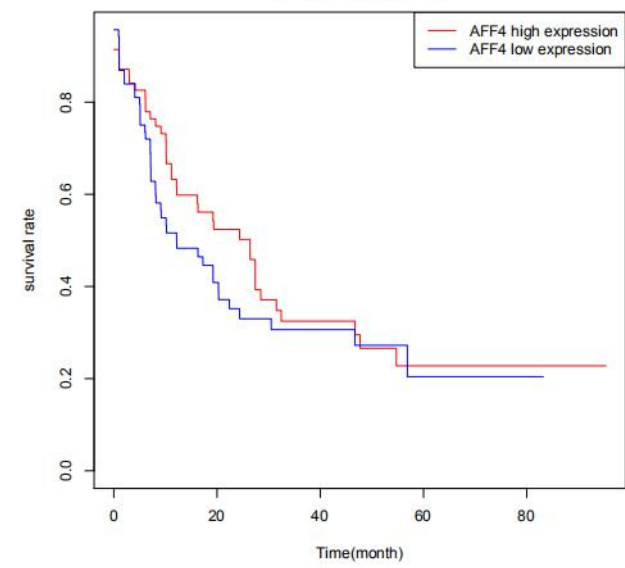

Figure 5 The prognostic values of AFFs expression in AML patients(TCGA).

(A) The overall survival of AFF1 expression in AML patients.

(B) The overall survival of AFF2 expression in AML patients.

(B) The overall survival of AFF1 expression in AML patients.

(D) The overall survival of AFF3 expression in AML patients.

\subsection{Related genes of AFFs in AML}

2.3.1 Correlation analysis of AFFs related genes in AML

The related genes of the AFFs family were obtained from the LinkedOmics database. The red dots in the volcano map show significantly positively related genes, and the green dots show significantly negatively related genes. The heat map shows the top 50 genes that are significantly positively correlated and negatively correlated with the AFFs family (Figure 6). Among them, KLHL11 (Pearson-Correlation=0.6296, $\mathrm{p}=1.71 \mathrm{E}-20$ ), MAPK8 (Pearson-Correlation $=0.5999, \mathrm{p}=2.741 \mathrm{E}-18$ ) and $\mathrm{ZNF} 81$ (Pearson-Correlation $=0.5952, \mathrm{p}=5.865 \mathrm{E}-18$ ) 
were significantly positively correlated with AFF1.MS4A3 (Pearson-Correlation=0.6916, $\mathrm{p}=5.981 \mathrm{E}-26)$, LPO (Pearson-Correlation=0.6425, $\mathrm{p}=1.59 \mathrm{E}-21$ ) and ELANE (Pearson-Correlation $=0.6264, p=3.063 \mathrm{E}-20$ ) were significantly positively correlated with AFF2. KIF17 (Pearson-

Correlation $=0.5905, \mathrm{p}=1.230 \mathrm{e}-17$ ), ANHAK2 (Pearson-Correlation=0.5698, $\mathrm{p}=2.811 \mathrm{e}-16$ ) and BLNK (Pearson-Correlation $=0.5674, \mathrm{p}=3.958 \mathrm{e}-16)$ were significantly positively correlated with AFF3. DMXL1 (Pearson-Correlation=0.7884, p=6.400e-38), FNIP1 (Pearson-Correlation=0.7770, $\mathrm{p}=3.350 \mathrm{e}-36$ ) and RICTOR (Pearson-Correlation $=0.7730, \mathrm{p}=1.246 \mathrm{e}-35$ ) were significantly positively
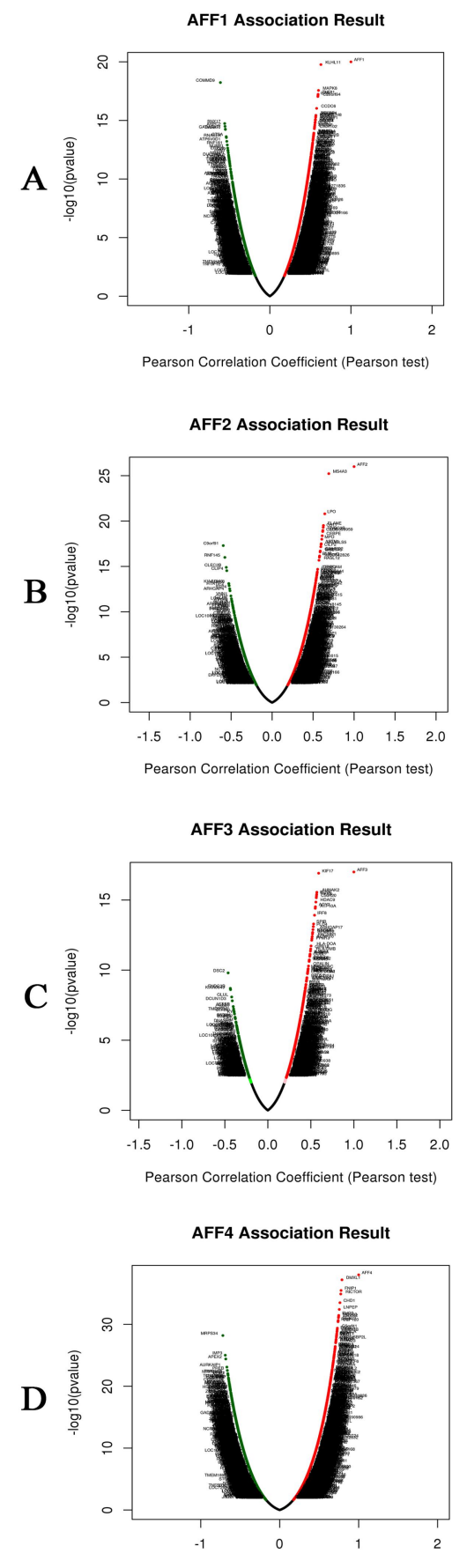
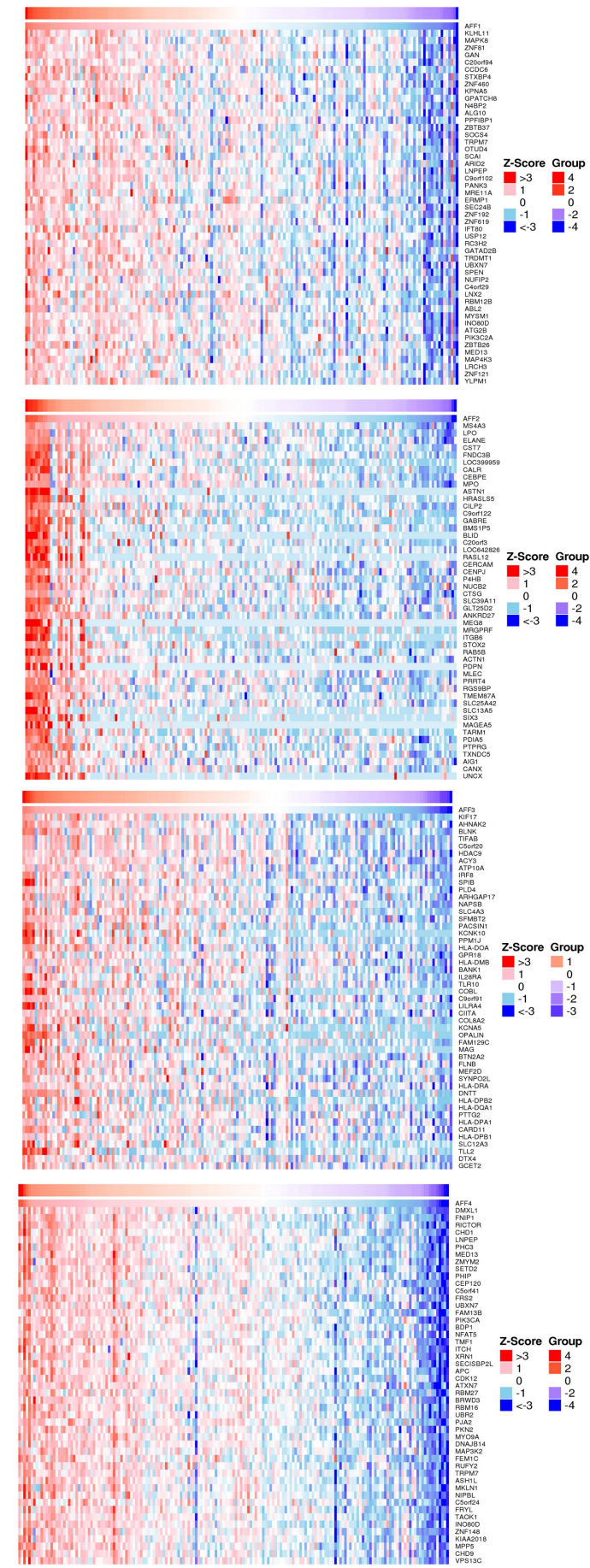
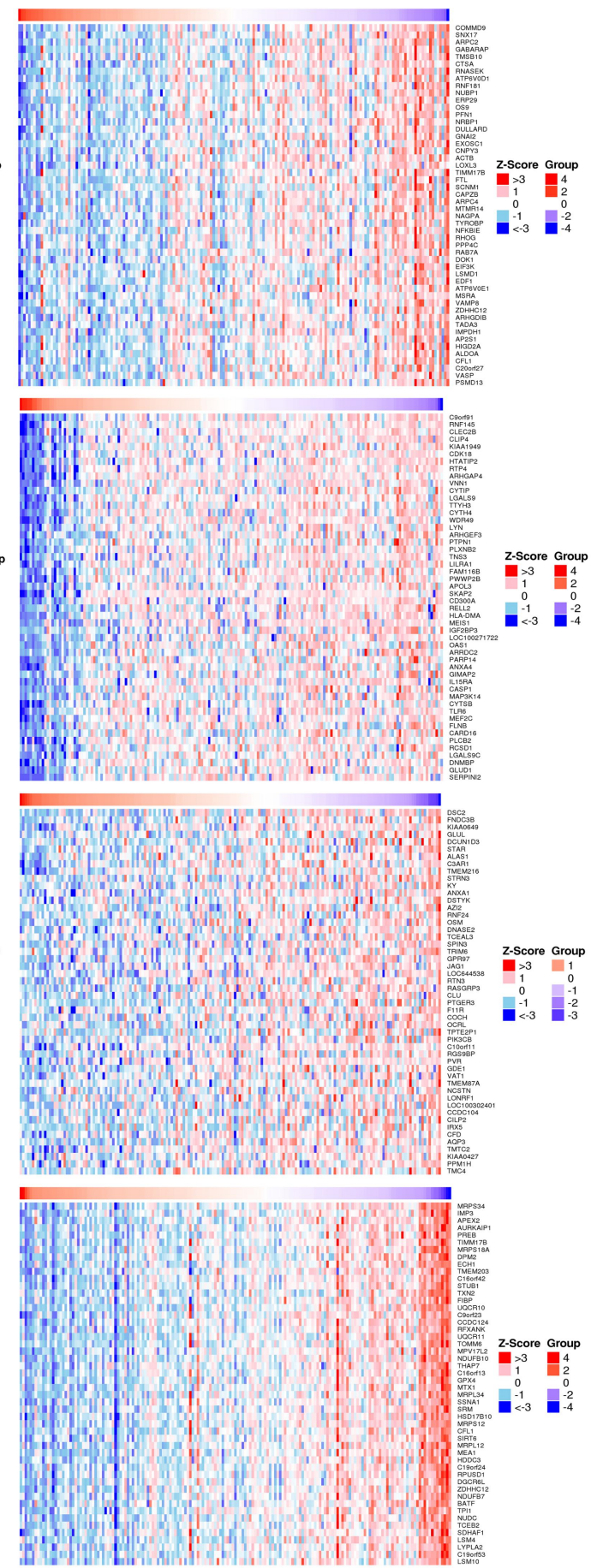
Figure 6 Differentially expressed genes correlated with AFFs in AML (LinkedOmics). (A,B,C,D) Volcano plots and heat maps indicating genes positively and negatively genes correlated with AFF1, AFF2, AFF3,
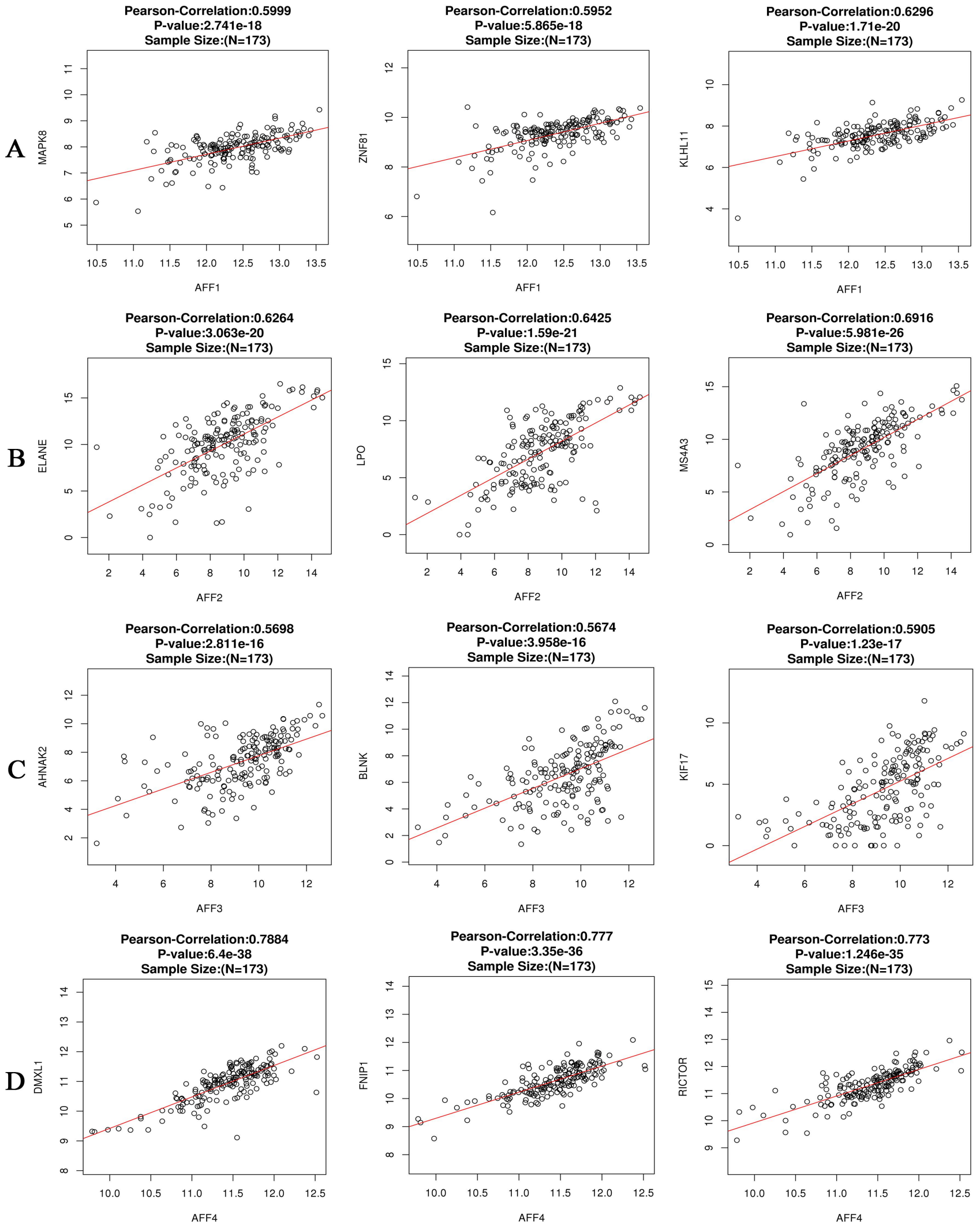
Figure 7 Gene correlation expression analysis for AFFs (LinkedOmics).

(A) The scatter plots show Pearson's correlation of AFF1 expression with expression of

KLHL11,MAPK8,ZNF81.(B) The scatter plots show Pearson's correlation of AFF2 expression with expression of MS4A3,LPO,ELANE. (C) The scatter plots show Pearson's correlation of AFF3 expression with expression of KIF17,AHNAK2,BLNK. (D) The scatter plots show Pearson's correlation of AFF4 expression with

\subsubsection{Enrichment analysis of AFFs related genes in AML}

The AFFs related genes obtained by LinkedOmics were used for functional enrichment analysis and KEGG signal pathway enrichment analysis (Figure 8). The main function is to regulate the stability of mRNA, the regulation of cell cycle, and the transcription of DNA. Signal pathway analysis speculates that AFFs may be involved in the regulation of proteoglycan pathways, cell adhesion molecules (CAMs), and Wnt signaling pathways in cancer.

A

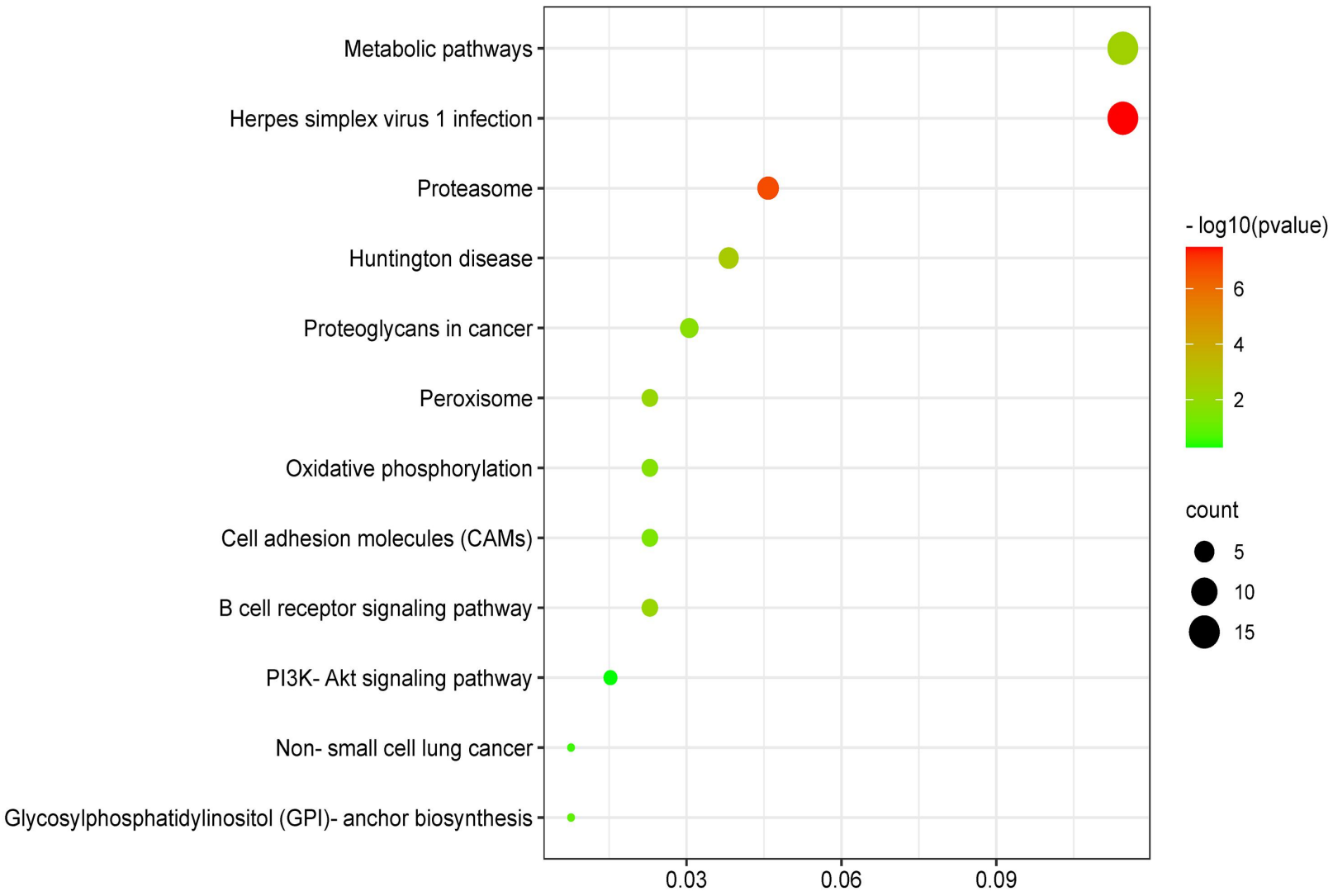




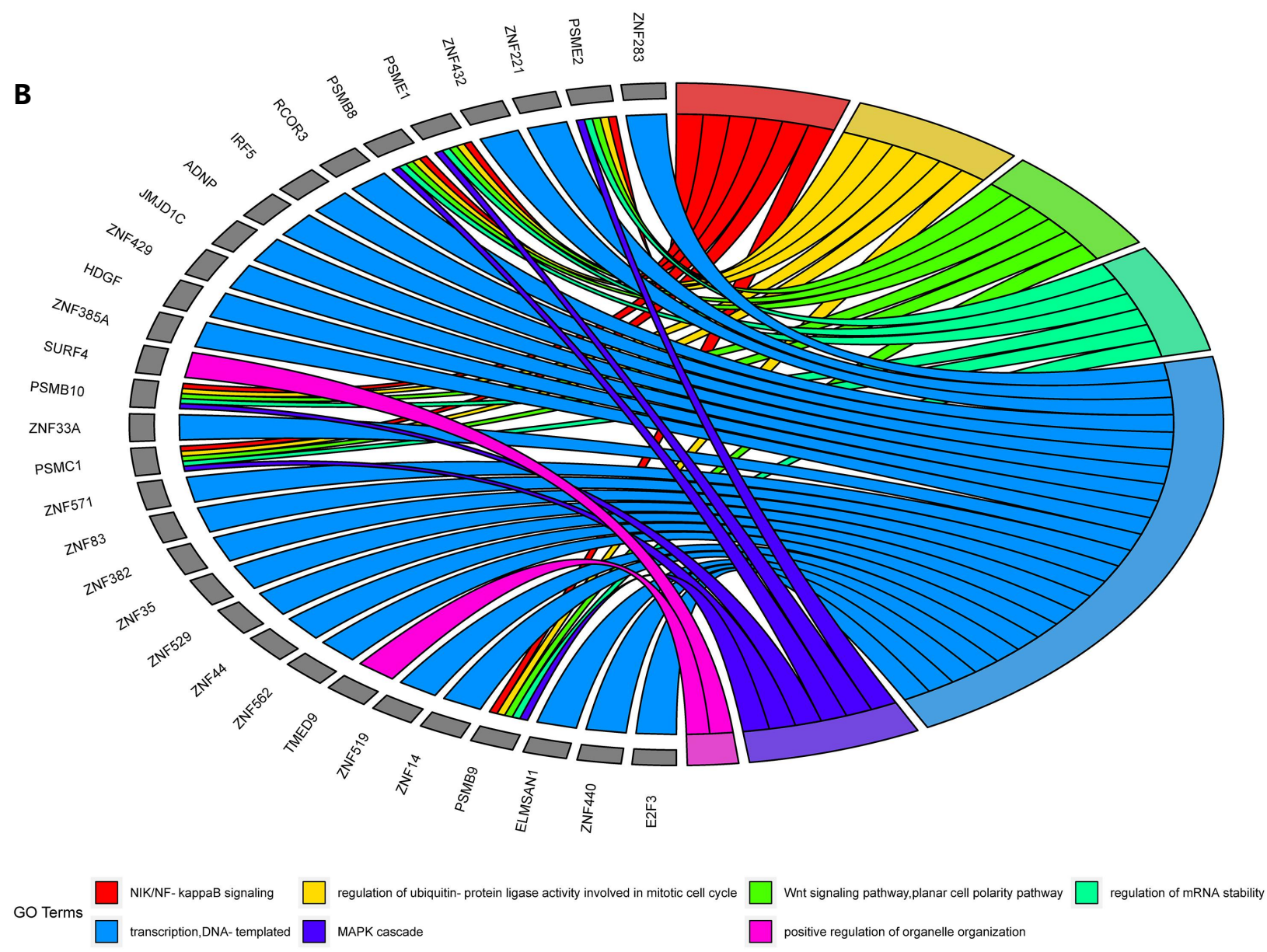

Figure 8 Functional enrichment analysis of AFFs

( A ) KEGG pathway analysis of AFFs(Kobas).

( B ) GO-BP terms of AFFs ( DAVID ).

\section{Discussion}

As a kind of RNA binding protein, AFFs' biological function involves various tumors, including leukemia especially in lymphoid leukemia,and there is a lack of research on the occurrence and development of AML. In order to explore the potential functions of AFFs in AML, we used an online bioinformatics database for analysis.

The analysis of Oncomine database and GEPIA database showed that AFF1, AFF2, and AFF3 weere highly expressed in AML. At the cellular level, we also confirmed that AFF1 and AFF4 were highly expressed through EMBL-EBI and 
CCLE database. This study further confirmed that AFF1, AFF2, AFF3, and AFF4 are highly expressed in AML (not M3) through clinical samples. The high expression of AFF1, AFF2, and AFF4 had statistically significant $(\mathrm{p}<0.05)$. The expression of AFF family genes varies in different databases, cell lines, and our clinical data. Among them,AFF1 waf highly expressed in all databases, cell lines and our clinical data.In addition, we found that the high expression of AFF1 wad significantly negatively correlated with OS. At the same time, we also found that the high expression of AFF2 was significantly positively correlated with OS. Then we analyzed the UALCAN database and showed that the expression of AFF1, AFF2 and AFF4 in each stage were significantly different from M3.AFF1 expression was significantly lower in M3 type than other types, and AFF2 expression was significantly higher than other types in M3 type.Previous studies had shown that the prognosis of M3 type in AML was better, and our survival analysis data includes M3 type. Therefore, the high expression of AFF1 is significantly negatively correlated with OS, and the high expression of AFF2 is significantly positively correlated with OS, which is consistent with clinical practice. In addition, we also used the LinkedOmics database to perform correlation analysis of AFFs-related genes. Among them, KLHL11 and MAPK8 were significantly positively correlated with AFF1, the function of them were correlated with apoptosis[23,24]. MS4A3, LPO, ELANE were significantly related to AFF2.They were involved in early bone marrow differentiation of human hematopoietic cells [25] and lipid peroxidation [26]. Finding AFFs-related genes in the LinkedOmics database, and further performed functional enrichment analysis and signal pathway analysis of related genes. The function of AFFs gene involved the stability of mRNA, transcription of DNA and the regulation of proteoglycan pathway in cancer, so AFFs were related to the occurrence and development of a variety of tumors. Studies reported that AFF1 and AFF4 became key regulators in the pathogenesis of leukemia through MLL [27], and AFF4 can also regulate tumor progression in cancer mice [28]. This was consistent with our research. In addition, AFFs genes may also be related to CAMs and proteoglycans in cancer. CAMs were involved in the metastasis and invasion of cancer cells [29]. proteoglycans, such as 
HSPG2[30], which can promote the metastasis and invasion of solid tumors. This was consistent with the promotion of tumor cell migration and invasion by AFFs. Studies also confirmed that AFF4 was involved in the invasion and migration of melanoma cells [31], lung adenocarcinoma (LUAD) cells [32],and head and neck squamous cell carcinoma (HNSCC) cells[33] . Studies showed that AFF4 regulates the self-renewal of bladder cancer stem cells[34]. Our research found that AFFs were involved in Wnt signal pathway and cell cycle regulation.The signal transduction of the Wnt/Fzd pathway was related to the maintenance of hematopoietic stem cells and the establishment of acute leukemia[35]. Wnt pathway regulated the apoptosis of leukemia cells in AML[36,37], proving that AFFs may be involved in the proliferation and apoptosis of tumor cells.

\section{4 conclusion}

In this study, we analyzed the expression and prognostic value of AFFs, and explored the biological events related to the progression of AML. The results showed that AFF1 and AFF2 were highly expressed in AML, the high expression of AFF1 was significantly negatively correlated with OS, and the high expression of AFF2 was significantly positively correlated with OS.

\section{Materials and methods}

\subsection{Bioinformatics methods}

\subsubsection{GEPIA database}

TCGA and GTEx have generated a large amount of RNA sequencing data. GEPIA provides key interactive and customizable functions based on TCGA and GTEx, including differential expression analysis, contour mapping, correlation analysis, similar gene detection and dimensionality reduction analysis[38]. Use GEPIA to analyze the expression changes of AFFs in AML samples and normal samples;

\subsubsection{ONCOMINE database}

The oncomine database has collected 18,000 genes, pathways, and networks in cancer gene expression microarrays, which span most cancer types and subtypes. The database aims to collect, standardize, analyze and provide cancer transcriptome data 
to the biomedical research community[39]. Use the oncomine database to compare the expression data of AFFs in AML and normal samples.

\subsubsection{LinkedOmics database}

The LinkedOmics database is a multi-omics database based on mass spectrometry (MS) global proteomics data generated by the Clinical Proteomics Tumor Analysis Association (CPTAC) on selected TCGA tumor samples. Contains multi-omics data and clinical data of 32 cancers and 11158 patients from the Cancer Genome Atlas (TCGA) project[40]. Analyze the related genes of AFFs through the LinkedOmics database

\subsubsection{Encyclopedia of Cancer Cell Lines(CCLE) database}

CCLE database includes gene expression, sequencing and single nucleotide polymorphism (SNP) array copy number of 1,650 genes in 947 cell lines[41]. The CCLE data was used to prove the expression of AFFs in cancer cell lines.

\subsubsection{The European Bioinformatics Institute (EMBL-EBI) database}

EMBL-EBI database maintained by the European Institute of Bioinformatics (EBI) collects nucleotide sequences and annotations available from public sources. In September 2006, the size of the EMBL nucleotide sequence database has increased from 58.7 million to 80.5 million. The database is part of international cooperation with DDBJ (Japan) and GenBank (USA)[42]. The goal is to collect and present nucleotide sequences and annotations as comprehensively as possible, and to further verify the expression of AFFs in AML cell lines.

\subsubsection{UALCAN database}

UALCAN database uses TCGA transcriptome and clinical patient data to study gene expression levels, not only comparing tumor and normal tissue samples, but also comparing tumors at different pathological stages, different grades and different races specialty.We compared the AFFs' difference expression level of different stages in AML by using the UALCAN database [43].

\subsubsection{TCGA database}

We downloaded the transcriptome data and clinical data of AML patients through the TCGA database, and converted the probe ID to the gene name according 
to the annotation file. We matched the patient's ID number with the corresponding RNA expression profile and clinical data, and excluded patients whose ID numbers cannot match. In the end, we selected 140 patients with complete clinical data for survival analysis. The survival curve was generated by running the $\mathrm{R}$ package "survival" using Rstudio ( version4.0.4 ), and the difference in survival curve was compared by log-rank test. A p value $<0.05$ is considered significant.

\subsubsection{DAVID and KOBAS database}

The DAVID database and the KOBAS database are based on the gene function categories in the gene list and the signal pathways involved in regulation. Classification can quickly help clarify new biological processes related to cell functions and pathways. GO analysis and KEGG analysis of AFFs-related genes were carried out through DAVID database and KOBAS database.

\subsection{Patients and specimens}

\subsubsection{Clinical specimens}

We collected 24 AML cases (non-M3 type) admitted to the Affiliated Hospital of Southwest Medical University as the experimental group, and 8 non-hematological malignancies (including anemia and thrombocytopenia) as the control group. The diagnostic criteria of AML patients refer to the WHO 2016 standard and are classified according to the FAB classification. The treatment of AML patients is mainly based on the Chinese Adult AML Diagnosis and Treatment Guidelines (non-APL) (2017)[44]. Collecting clinical data such as patient age, gender, gene mutation/fusion, subtype classification, risk, complete remission (CR), etc.

\subsubsection{Real-time fluorescent quantitative PCR (RT-qPCR)}

Total RNA was extracted using RNAsimple Total RNA Kit (TIANGEN, China, Beijing). cDNA was synthesized using TransScript All-in-One First-Strand cDNA Synthesis SuperMix for qPCR kit (TransGen Biotech, China, Beijing), and PerfectStart Green qPCR SuperMix (Transgen, China, Beijing) was used to detect AFFs mRNA levels. The primers sequences were AFF1- forward,5'-GCTTC TCTGGGGTTTGTTCA-3', AFF1-reverse, 5'-AAGGAACGTCATCCATGCT C-3', AFF2- forward, 5'-TGGAGAGGGTATCTGTGCGA-3', AFF2- reverse, 
5'-AAGTCCCTTGGCTTCGGATG-3',AFF3- forward ,5'-CAGGTTTGGGAACTC CAACG-3', AFF3- reverse, 5'-CTCAACAGGATGATGGCACG-3', AFF4- forward , 5'-AAGAGGCGGGTGACACTTTA-3', AFF4- reverse,5'-GCAAATGAGCCATCC CAGAG-3', $\beta$-Actin-forward,5'-GGCGGCACCACCATGTACCC-3', $\beta$-Actin-reverse, 5'-CCACACGGAGTACTTGCGC-3'. The 2- $\Delta \Delta \mathrm{CT}$ method was used to calculate the relative mRNA expression.

\subsection{3 statistical analysis}

Statistical analysis was performed using SPSS (version 17.0, IBM Corp. Armonk, NY) and GraphPad Prism (version 8.0, GraphPad Software). Student's t-test was used to test the expression difference between the experimental group and the control group. According to the median value of AFFs expression, patients were divided into high expression group and low expression group, and the difference between high expression group and low expression group was statistically compared by Fisher's exact test analysis. When the $\mathrm{P}$ value is less than 0.05 , it is considered statistically significant.

\section{Acknowledgements}

The authors gratefully acknowledge GEPIA database,ONCOMINE database,LinkedOmics database,Encyclopedia of Cancer Cell Lines(CCLE) database,the European Bioinformatics Institute (EMBL-EBI) database,UALCAN database,TCGA database and DAVID and KOBAS database, which made the data available

\section{Authors' contributions}

$\mathrm{XC}, \mathrm{QY}, \mathrm{XQ}, \mathrm{JL}, \mathrm{YZ}, \mathrm{SLL}$ and WJL are the principle investigator. XC and QY completed specimen collection and data processing.XC and SLL designed experiments.XC, QY, XQ, JL, YZ, SLL and WJL participated in the writing and revision of the article.All authors read and approved the final manuscript.

\section{Funding}

This work was funded by the Basic Research Project of Sichuan Province (No. 
2019YJ0690); The Major Science and Technology Projects in Sichuan Province (No. 2019YFS0531).

\section{Availability of data and materials}

The raw data of this study are derived from the GEPIA database (http://gepia.cancer-pku.cn/), oncomine database (http://www.oncomine.org), LinkedOmics database (http://www.linkedomics.org), CCLE database (http://www.broadinstitute.org/ccle/home), $\quad$ EMBL-EBI database (http://www.ebi.ac.uk/embl), UALCAN (http://ualcan.path.uab.edu), TCGA database (https://portal.gdc.cancer.gov), DAVID database

(https://david.ncifcrf.gov/summary.jsp) and the KOBAS database (http://kobas.cbi.pku.edu.cn).

\section{Ethics approval and consent to participate}

This study was conducted in accordance with a set of principles of the Declaration of Helsinki and was approved by the Medical Ethics Committee of the Affiliated Hospital of Southwest Medical University.

\section{Consent for publication}

Not applicable

\section{Competing interests}

The authors declare that they have no competing interests. 


\section{References}

1. Qian L, Wei L, Ying Z,et al. PRKD2 Promotes Progression and Chemoresistance of AML via Regulating Notch1 Pathway [J].Onco Targets Ther. 2019,12:10931-10941. doi: 10.2147/OTT.S233234.eCollection 2019.

2. Yasser H E, Ragia H B, Fatma A E et al. IDH Mutations in AML Patients; A higher Association with Intermediate Risk Cytogenetics [J].Asian Pac J Cancer Prev. 2020;21 (3):721-725. doi: 10.31557/APJCP.2020.21.3.721.

3. De Kouchkovsky I,Abdul-Hay M.'Acute myeloid leukemia: a comprehensive review an d 2016 update'[J]. Blood Cancer J. 2016;6(7):e441.doi: 10.1038/bcj.2016.50.

4. Li T, Xiaoping W, Lixia Z,et al.Amplified HMGA2 promotes cell growth by regulatin g Akt pathway in AML [J]. J Cancer Res Clin Oncol. 2015,142(2):389-99.doi: 10.100 7/s00432-015-2036-9. Epub 2015 Aug 30.

5. Xiao-Qing Y, Dao-Yu Z, Han Y et al.Evaluation of DNMT3A genetic polymorphisms as outcome predictors in AML patients [J].Oncotarget. 2016,7(37):60555-60574.doi: 10. 18632/oncotarget.11143.

6. Dengyang Z, Yao G, Yuming $Z$ et al.Expression of a recombinant FLT3 ligand and it s emtansine conjugate as a therapeutic candidate against acute myeloid leukemia cells with FLT3 expression [J].Microb Cell Fact. 202,20(1):67.doi: 10.1186/s12934-021-015 $59-6$.

7. Döhner H, Weisdorf DJ, Bloomfield CD.Acute Myeloid Leukemia [J].N Engl J Med . 2015,373(12):1136-52.doi: 10.1056/NEJMra1406184.

8. Mireille M, Dominique D, Mounia B, et al.Functional characterization of the AFF (AF4 /FMR2) family of RNA-binding proteins: insights into the molecular pathology of FR AXE intellectual disability[J].Hum Mol Genet. 2011,20(10):1873-85.doi: 10.1093/hmg/d dr069.Epub 2011 Feb 17.

9. Fateen Farhana I, Rahman J, Saiful Effendi S,et al.MicroRNA-200c and microRNA-31 regulate proliferation, colony formation, migration and invasion in serous ovarian canc er [J].J Ovarian Res.2015,8:56.doi:10.1186/s13048-015-0186-7.

10. Junjie $\mathrm{Y}$, Qian D, Shaohua $H$ et al.Suppression of the NTS-CPS1 regulatory axis by AFF1 in lung adenocarcinoma cells [J].J Biol Chem. 2021,296:100319.doi: 10.1016/j.jb 
c.2021.100319

11. Tiziana F, Armando C, Mariateresa Z, et al.Crosstalk between 14-3-30 and AF4 enhan ces MLL-AF4 activity and promotes leukemia cell proliferation [J].Cell Oncol (Dordr). 2019,42(6):829-845.doi: 10.1007/s13402-019-00468-6.

12. Sneha T, Mary S, Scott D, et al.First report of t $(5 ; 11)$ KMT2A-MAML1 fusion in de $n$ ovo infant acute lymphoblastic leukemia [J].Cancer Genet.2020,248-249:31-33.doi: 10.1 016/j.cancergen.2020.09.004.

13. Qingjun W, Xuanmei L, Hexin L,et al.Identification of differentially expressed circular RNAs associated with thymoma [J].Thorac Cancer. 2021.doi: 10.1111/1759-7714.1387 3.

14. Jessica L B, Sven H, Jessica $\mathrm{K} \mathrm{H}$,et al.Identification of RNA-Binding Proteins as Tar getable Putative Oncogenes in Neuroblastoma [J].Int J Mol Sci .2020,21(14):5098.doi: 10.3390/ijms21145098.

15. Justin A B, Jeffrey G, Claire P,et al.Nonkeratinizing Squamous Cell Carcinoma of the Sinonasal Tract With DEK-AFF2: Further Solidifying an Emerging Entity [J].Am J S urg Pathol. 2021,45(5):718-720.doi: 10.1097/PAS.0000000000001596.

16. Xuefeng B, Zhengwei C, Anwei $\mathrm{Z}$ et al.Circular RNA CircAFF2 Accelerates Gastric Cancer Development by Activating MiR-6894-5p and Regulating ANTXR 1 Expressio n[J].Clin Res Hepatol Gastroenterol. 2021,101671. doi: 10.1016/j.clinre.2021.101671.

17. Fan Z, Dongjiao W, Wei W,et al.Comprehensive profiling of circular RNA expression $\mathrm{s}$ reveals potential diagnostic and prognostic biomarkers in multiple myeloma [J].BMC Cancer. 2020,20(1):40.doi: 10.1186/s12885-020-6515-2.

18. Rachael $\mathrm{J}$ L T, Laura $\mathrm{J} \mathrm{G}$, Catherine P,et al.Investigation of rheumatoid arthritis susce ptibility genes identifies association of AFF3 and CD226 variants with response to an ti-tumour necrosis factor treatment [J].Ann Rheum Dis. 2010,69(6):1029-35.doi:10.1136 /ard.2009.118406.

19. L Lefèvre,H Omeiri,L Drougat,, et al.Combined transcriptome studies identify AFF3 as a mediator of the oncogenic effects of $\beta$-catenin in adrenocortical carcinoma [J].Onco genesis. 2015,4(7):e161.doi: 10.1038/oncsis.2015.20.

20. Yawei S, Yang Z, Yunjian Z,et al.AFF3 upregulation mediates tamoxifen resistance in 
breast cancers [J].J Exp Clin Cancer Res. 2018,37(1):254.doi: 10.1186/s13046-018-09 $28-7$.

21. Qingyue X, Yuning Z, Xingying Q,et al.AFF4 regulates osteogenic differentiation of $h$ uman dental follicle cells [J].Int J Oral Sci. 2020,12(1):20.doi: 10.1038/s41368-020-008 $3-9$.

22. Chengqi L , Edwin R S, Hidehisa T, et al.AFF4, a component of the ELL/P-TEFb el ongation complex and a shared subunit of MLL chimeras, can link transcription elong ation to leukemia [J].Mol Cell. 2010,37(3):429-37.doi: 10.1016/j.molcel.2010.01.026.

23. Bajinder S D, Tiziana C, Akhila G S,et al.Update on the Kelch-like (KLHL) gene fa mily [J].Hum Genomics. 2013,7(1):13.doi: 10.1186/1479-7364-7-13.

24. Xu P, Zhang G, Hou S,et al.MAPK8 mediates resistance to temozolomide and apopto sisof glioblastoma cells through MAPK signaling pathway[J].Biomed Pharmacother.201 8;106:1419-1427. doi: 10.1016/j.biopha.2018.06.084.

25. Tomohiko I, Takafumi Y, Yusuke S,et al.Identification of MS4A3 as a reliable marke $r$ for early myeloid differentiation in human hematopoiesis [J].Biochem Biophys Res Commun.2018,495(3):2338-2343.doi: 10.1016/j.bbrc.2017.12.117.

26. Akshay A, Pawan K G, Sudesh P,et al.Analysis of smoking and LPO in ALS [J].Neu rochem Int. 2014,71:47-55.doi: 10.1016/j.neuint.2014.04.004.

27. Lokken AA, Achille NJ, Ming-Jin C,et al. Importance of a Specific Amino Acid Pairi ng for Murine MLL Leukemias Driven by MLLT1/3 or AFF1/4 [J].Leuk Res. 2014;3 8(11):1309-15. doi: 10.1016/j.leukres.2014.08.010.

28. Qian G, Jin Z, Zegui N, et al.The m6A Methylation-Regulated AFF4 Promotes Self-R enewal of Bladder Cancer Stem Cells [J].Stem Cells Int.2020,2020:8849218.doi: 10.11 55/2020/8849218.eCollection 2020.

29. Michalina J, Marina CP, Tina I.Cell adhesion in cancer: Beyond the migration of sin gle cells.J Biol Chem .2020;295(8):2495-2505.doi: 10.1074/jbc.REV119.007759.

30. Xiaojia Z, Simin L, Qian Z,et al.HSPG2 overexpression independently predicts poor survival in patients with acute myeloid leukemia[J].Cell Death Dis. 2020;11(6):492.doi: $10.1038 / \mathrm{s} 41419-020-2694-7$.

31. Hongyan H, Yi Z, Liufang Z,et al.AFF4 facilitates melanoma cell progression by regu 
lating c-Jun activity [J].Exp Cell Res. 2021,399(2):112445.doi: 10.1016/j.yexcr.2020.112 445.

32. Juan L, Jun G, Ye Y, et al.Long noncoding RNA ZFPM2 - AS1 is involved in lunga denocarcinoma via miR - 511 - 3p/AFF4 pathway [J].J Cell Biochem.2020,121(3):25342542.doi: $10.1002 / \mathrm{jcb} .29476$.

33. Peng D, Jiongke $\mathrm{W}$, Xuefeng $\mathrm{Z}$, et al.AFF4 promotes tumorigenesis and tumor-initiatio n capacity of head and neck squamous cell carcinoma cells by regulating SOX2 [J].C arcinogenesis.2018,39(7):937-947.doi: 10.1093/carcin/bgy046.

34. Qian G, Jin Z, Zegui N, et al.The m6A Methylation-Regulated AFF4 Promotes Self-R enewal of Bladder Cancer Stem Cells [J].Stem Cells Int.2020,2020:8849218.doi: 10.11 55/2020/8849218.eCollection 2020.

35. Cassaro A, Grillo G, Notaro M,et al.FZD6 triggers Wnt-signalling driven by WNT10BIVS1 expression and highlights new targets in T-cell acute lymphoblastic leukemia[J].Hematol Oncol . 2021.doi: 10.1002/hon.2840. Online ahead of print.

36. Takam Kamga P, Bazzoni R, Dal Collo G,et al. The Role of Notch and Wnt Signali ng in MSC Communication in Normal and Leukemic Bone Marrow Niche[J].Front Ce 11 Dev Biol . 2021 ;8:599276. doi: 10.3389/fcell.2020.599276. eCollection 2020.

37. Wakabayashi R,Hattori Y,Hosogi S,et al.A novel dipeptide type inhibitor of the Wnt $/ \beta$ -catenin pathway suppresses proliferation of acute myelogenous leukemia cells[J].Bioch em Biophys Res Commun . 2021;535:73-79.doi: 10.1016/j.bbrc.2020.12.027.

38. Zefang T, Chenwei L, Boxi K,et al.GEPIA: a web server for cancer and normal gene expression profiling and interactive analyses [J].Nucleic Acids Res.2017,45(W1):W98W102.doi: $10.1093 / \mathrm{nar} / \mathrm{gkx} 247$.

39. Daniel R R, Shanker K S, Vasudeva M,et al.Oncomine 3.0_ genes, pathways, and net works in a collection of 18,000 cancer gene expression profiles [J].Neoplasia.2007,9(2): 166-80.doi: $10.1593 /$ neo.07112.

40. Suhas V V, Peter S, Jing W,et al.LinkedOmics: analyzing multi-omics data within an d across 32 cancer types [J].Nucleic Acids Res.2018,46(D1):D956-D963.doi: 10.1093/n ar/gkx1090.

41. Ghandi M, Huang FW, Jané-Valbuena J, et al.Next-generation characterization of the Cancer 
Cell Line Encyclopedia[J].Nature.2019;569(7757):503-508.doi: 10.1038/s41586-019-1186-3.

42. Kulikova T, Akhtar R, Aldebert P, et al.EMBL Nucleotide Sequence Database in 2006 [J].Nucleic Acids Res. 2007;35(Database issue):D16-20. doi: 10.1093/nar/gk1913..

43. Chandrashekar DS, Bashel B, Balasubramanya SAH, et al.UALCAN: A Portal for Faci litating Tumor Subgroup Gene Expression and Survival Analyses[J].Neoplasia. 2017 ; 9(8):649-658. doi: 10.1016/j.neo.2017.05.002.

44. Dohner H, Estey E, Grimwade D, Amadori S, Appelbaum FR, Buchner T, et al. Dia gnosis and management of AML in adults: 2017 ELN recommendations from an inter national expert panel. Blood. 2017;129(4):424-47. 10.1182/blood-2016-08-733196. 


